\title{
Insights from macroevolutionary modelling and ancestral state reconstruction into the radiation and historical dietary ecology of Lemuriformes (Primates, Mammalia)
}

Ethan Fulwood ( $\nabla$ ethanfulwood@upike.edu )

University of Pikeville Kentucky College of Osteopathic Medicine https://orcid.org/0000-0002-73538448

Shan Shan

Duke University Julia Winchester

Duke University Henry Kirveslahti Duke University

Robert Ravier

Duke University

Shahar Kovalsky

Duke University

Ingrid Daubechies

Duke University

Doug Boyer

Duke University

Research article

Keywords: Adaptive radiation, macroevolution, paleobiology

Posted Date: December 18th, 2020

DOl: https://doi.org/10.21203/rs.3.rs-130245/v1

License: (c) (1) This work is licensed under a Creative Commons Attribution 4.0 International License. Read Full License 
Version of Record: A version of this preprint was published at BMC Ecology and Evolution on April 21st, 2021. See the published version at https://doi.org/10.1186/s12862-021-01793-x. 


\section{Abstract}

The lemurs are a highly species-rich clade of primates, which, confined almost entirely to the island of Madagascar, have evolved to rival the diversity of rest of the primate order. We test the adaptive radiation model of Malagasy lemur diversity using a novel combination of phylogenetic comparative methods and geometric methods for quantifying tooth shape. We apply macroevolutionary model fitting approaches and disparity through time analysis to dental topography metrics associated with dietary adaptation, an aspect of mammalian ecology which appears to closely related to diversification in many clades. Metrics were also reconstructed at internal nodes of the lemur tree and these reconstructions were combined to generate dietary classification probabilities at internal nodes using discriminant function analysis. We used these reconstructions to calculate rates of transition toward folivory per million-year intervals. Finally, lower second molar shape was reconstructed at internal nodes by modelling the change in shape of 3D meshes using squared change parsimony along the branches of the lemur tree. Our analyses of dental topography metrics do not recover an early burst in rates of change or a pattern of early partitioning of subclade disparity. However, rates of change in adaptations for folivory were highest during the Oligocene, an interval of possible forest expansion on the island. Reconstruction of the molar morphologies corresponding to the ancestral nodes of the lemur tree suggest that this may have been driven by a shift toward defended plant resources.

\section{Background}

The lemurs of Madagascar account for a quarter of global primate diversity, and, when recently extinct forms are considered, occupy ranges of body size, locomotor style, and dietary niche comparable to the diversity observed among all other living primates $(1-3)$. The taxonomic and ecological diversity of lemurs greatly exceeds that of the relatively narrowly adapted Lorisiformes, which evolved in the presence of anthropoid primates on continental Africa and Asia (4). This diversity has prompted researchers to hypothesize that lemurs evolved on Madagascar through a process of adaptive radiation (5-10). In this paper, predictions of an adaptive radiation model are investigated with regard to dietary adaptation using approaches that combine phylogenetic methods with the quantification of molar shape evolution.

\section{Adaptive radiation}

The "Law of adaptive radiation" was formulated by Osborn (11) to explain the repeated evolution of mammal clades with similar ecological breadths and patterns of niche occupation on different landmasses. Simpson $(12,13)$ reconceptualized adaptive radiation as a process of zonal differentiation within an evolving clade as it explores a landscape of adaptive peaks previously unoccupied, or occupied by competitively inferior groups. He predicted that high rates of ecomorphological evolution will occur within groups as newly formed subclades traverse the ecological distances separating adaptive peaks. These peaks become available to evolving subclades through events that increase available ecological space. Classic examples include dispersal events, the extinction of antagonist clades, and the acquisition of "key innovations" (13-18). Ecological opportunity may also result from the environmentally mediated opening 
of suitable niche space (18). In primates, this is likely to involve the spread of tropical or paratropical forests, to which primate diversity is strongly linked (19).

\section{Lemur Evolution}

Madagascar has been separated from continental Africa since the Jurassic ( $136 \mathrm{Ma})$, long before the plausible origins of crown strepsirrhines (20-23). The long separation of Madagascar from Africa appears to necessitate at least one overwater dispersal coincident with the origins of Lemuriformes, likely on floating rafts of vegetation, as first proposed by Millot (24). Modern prevailing currents transport debris discharged from African rivers north or south along the African coast and away from Madagascar, but this pattern of currents was only established during the Oligocene and early Miocene by the disruption of subtropical gyres created by the northward tectonic movements of Madagascar and Australia (21). During most of the Paleogene, reconstructed paleocurrents would have been more amenable than they are today to dispersal from the east African coast to western Madagascar, which is reflected in the high percentage of dispersal-limited (non-volant and non-swimming) taxa that appear to have arrived on the island during this interval $(22,23)$. This list of taxa includes lemurs, which are often thought to have been among the first arrivals, during the Paleocene or early Eocene $(5,22,25,26)$.

The hypothesized date for the arrival of lemurs on Madagascar is based on the estimated divergence of Daubentonia from the other lemurs (25). Molecular clock estimates have returned highest probability estimates for this date between 66 and $46 \mathrm{Ma}$, encompassing the Paleocene and Eocene $(25,27-33)$, with more recent studies generally favoring an Eocene divergence $(28,29,33)$. Recent reexamination of the fossil taxa Propotto and Plesiopithecus, from the Miocene of Kenya and Eocene of Egypt respectively, has postulated that these taxa should be placed on the stem of Daubentonia (a grouping termed Chiromyiformes), complicating the traditional understanding of lemur biogeography (34). Ancestral biogeographic reconstructions incorporating this new topology support a mainland African origin for lemurs, but two independent dispersals. This revised chronology decouples estimates of the date of the earliest dispersal of the non-chiromyiform lemurs (Lemuriformes) from the basal divergence of Daubentonia. Revised estimates suggest that the dispersal of Lemuriformes may have occurred as late as the Miocene, although in the absence of contradicting information, it seems likely that it must have preceded the change in current direction initiated by the northward drift of Madagascar, which likely would have occurred by the end of the Oligocene (21). The tree topology proposed by Gunnell et al. (34) would also imply that the unusual ecological role of Daubentonia evolved while its lineage was still on the African mainland, limiting the bounds of any putative Malagasy adaptive radiation to Lemuriformes.

\section{Predictions of an adaptive radiation model}

Cladogenesis is thought to be associated with the opening exploitation of newly available ecological opportunity (13). The sweepstakes dispersal of lemuriforms to Madagascar is often linked to the diversification of lemurs for this reason (23-25). The tectonic history of Madagascar may have also been important in mediating lemuriform ecological opportunity, however. Madagascar reached its southern terminus during the middle Cretaceous, and has moved north on a path through the global aridity belt 
formed by the circulation of Hadley cells at approximately 40-30 S latitude, likely substantially affecting climate conditions on the island $(20,21,35)$. Passage beyond the desert belt and into the influence of seasonal monsoons likely increased precipitation that drove the spread of more mesic environments, including forests, across the island during the Eocene and Oligocene $(20,22,35)$. The Eocene-Oligocene transition is also associated with widespread global cooling and drying that seems to have contributed to major extinctions of primates on northern hemisphere continents $(4,36,37)$. Comparisons between the extant mammalian faunas of Madagascar the Paleogene faunas of mainland Africa suggest that this event may have had a winnowing effect on Madagascar as well (10). The interaction of this global climate pattern with the local effects of tectonic movements may have created unique conditions on Madagascar spurring the diversification of lemurs during this interval.

Lemurs are sorted into speciose, apparently ecologically differentiated clades of relatively ancient origin, but most lemur species appear to have diverged only within the last few million years. Explanations of this pattern have focused the repeated development of dispersal barriers as the landscape of Madagascar evolved across the Pleistocene (5,38-40). Landscape models do not address the deeper adaptive differentiation of lemur genera and families predicted by an adaptive radiation deep in the history of Lemuriformes, however. Herrera (9) examined the dynamics of taxonomic and phenotypic body size diversification in lemurs using a series of phylogenetic model fitting approaches. He found evidence for accelerating rates of speciation toward the tips of the tree, supporting the suggestions of lemur diversification models that emphasize geologically recent speciation events to explain the taxonomic diversity of the extant fauna (e.g. 40). Godfrey et al. (10) revisit this diversification modelling approach, using a broader set of metrics and examining the topologies recovered by both Kistler et al. (28) and Herrera and Dávalos (29), and find no clear evidence for either gradually increasing rates of diversification or a mass extinction followed by a rapid diversification.

The near absence of a terrestrial Tertiary fossil record on Madagascar hampers our ability to reconstruct macroevolutionary diversification dynamics across deep time on the island $(5,9)$. It is particularly challenging to reconstruct the pattern of species diversification in the absence of fossil evidence $(9,41)$. Adaptive radiation is expected to generate high rates of speciation early in clade history which will decline as clades diversify and fill available niches $(14-16,18,42)$. However, adaptive radiation may also involve high rates of extinction in populations morphologically intermediate between adaptive peaks, obscuring the signal of elevated speciation in the resulting branching diversification rate (43). Many lemur species are relatively recently diverging, which may indicate iterative extinctions and diversifications of ecologically analogous taxa accompanying climate cycles across the Neogene and Quaternary, as suggested by Martin (5).

An adaptive radiation model for the origins of high-level lemur diversity would predict increased rates of ecomorphological evolution during one or both of the hypothesized periods of ecological opportunity (early Eocene or Oligocene), however, which should be tractable even in the context of high levels of species extinction. Herrera (9) found evidence that rates of body mass showed a trended decrease after the origin of the clade, as best fit by an "early burst" model of evolution (43). The body masses of taxa 
occupying different macroevolutionary niches (defined by activity pattern and diet) also showed separate evolutionary optima in a multi-peak OU model. These patterns are consistent with a partitioning into macroevolutionary niches early in the diversification of major lemur families. They suggest that at least some aspects of lemur ecology may have evolved through a pattern consistent with an adaptive radiation linked to the dispersal of lemurs to Madagascar and the origins of the lemur clade (44).

We test the predictions of the adaptive radiation model of lemur evolution using dietary ecomorphology. Although not the only aspect of lemur ecology hypothesized to have evolved by adaptive radiation (6), diet represents an important axis of high-level niche partitioning during adaptive radiation $(13,45,46)$. If the high-level dietary diversity of lemurs has been shaped by a phase of general adaptive radiation, then A) High rates of adaptive evolution are expected during periods of ecological opportunity; and B) Partitioning of ecomorphological disparity is expected to occur during these intervals of ecological opportunity. We test these predictions by phylogenetically modeling the evolution of dental topography metrics, a class of tooth shape descriptors; and reconstructing the ancestral shapes of whole teeth at internal nodes of lemuriform phylogenetic trees.

\section{Results}

\section{Model fitting}

When considering all lemurs (lemuriformes and chiromyiformes), interspecific variance in the dental topography metrics DNE and RFI was best fit by a BM model of evolution (DNE: $65 \%$ of model weight; RFI: $64 \%$ of model weight). BM was still the best model when considering only groups branching after the beginning of the Oligocene (i.e. with chiromyiforms and Megaladapis pruned) (DNE: $49 \%$ of model weight; RFI: $62 \%$ of model weight). DNE CV was best fit by an OU model (Lemurs: $91 \%$ of model weight; Oligocene lemurs: $62 \%$ of model weight). No fits preferred an EB process (Supplemental Table 3).

\section{Disparity through time}

A lineage evolving through adaptive radiation should show a significantly negative MDI, indicating the accumulation of large proportion of total clade disparity early in the divergence of major subclades (47). MDI was negative but not significantly less than zero in the total lemuriform clade (MDI $=-0.10 ; p=0.48$ ) (Fig. 1) and positive in the clade of lemurs diverging in the Oligocene (MDI $=0.25 ; p=0.95$ ).

\section{Rates of evolution through time}

Rates of evolution toward combinations of dental topography metrics indicative of folivory calculated using mean reconstructions (MR) visually peak during the Oligocene, coinciding with the hypothesized spread of modern Malagasy forests as the island moved into moister, tropical latitudes (Fig. 2). This is confirmed by modelling approaches. Rates of evolution were higher during the Oligocene in models using both MRs and posterior distributions of reconstructions (PDRs) (coefficients from the model indicating the effect of a branch crossing the Oligocene interval $=0.64 ; 0.19)$. Rates of evolution appear to be 
relatively low during the Eocene, contrary to the expectations of an Eocene dispersal model, which is confirmed by modeled comparisons of rates of evolution during and after the first 10 million years of the basal divergence of lemurs (coefficients $=-0.18 ;-0.29$ ) (Supplemental Table 4; Fig. 2).

\section{Ancestral tooth shapes}

The morphologies of reconstructed teeth are described in detail in the supplemental material (Supplemental Fig. 2-6). Ancestral lorisiformes and lemuriforms are broadly similar in morphology, and both resembled their last common ancestor. A major divergence occurs at the last common ancestor of the "large-bodied" lemurs, (indriidae + lemuridae), which exhibits a more bilophodont morphology (Fig. 3).

Plotting ancestral state reconstructions of ariaDNE and ariaDNE CV values reveals interesting patterns (Fig. 4). As might be expected under a BM model of evolution the most basal nodes cluster close together. This pattern of low variance is maintained over the first 20 million years of strepsirrhine history. From $40-30 \mathrm{Ma}$, ancestral lorisiform and lemuriform morphotypes strongly diverge, with lorisiforms (node 74) moving into a space of high ariaDNE and ariaDNE CV and lemuriforms (node 52) into a space of relatively low values for both metrics. From 30-20 Ma, the ancestral large-bodied (indriid + lemurid) node (node 59) diverges from those of cheirogaleids and lepilemurids. After $20 \mathrm{Ma}$, the ancestral indriids move into a mostly distinctive morphospace away from the rest of the lemurs. Lemurids expand to occupy much of the relatively low ariaDNE region of lemur morphospace as they diversify over this interval.

\section{Discussion}

Simpsonian adaptive radiation occurs through the "more or less simultaneous divergence of numerous lines all from much the same ancestral adaptive type into different, also diverging adaptive zones" (13). The results of our study do not support the hypothesis that the diversity in diet of the lemuriform clade emerged as a product of Simpsonian style adaptive radiation at the beginning of the Eocene, either as a response to dispersal to Madagascar or as a signal of cladogenesis. There is no signal of elevated rates of evolution in any of the three dental topography metrics, no early partitioning of morphological disparity, and relatively low rates of adaptive evolution away from the ancestral frugivorous ecology and toward folivory. Evidence for a dietary adaptive radiation of lemurs during the Oligocene is mixed. High rates of transition toward folivory are observed during the first $10 \mathrm{Ma}$ of this interval, which is consistent with increased exploitation of defended plant resources in expanding and diversifying forests. However, evolutionary modelling and MDI fail to indicate rapidly divergent evolution into distinct ecological niches, at least as captured by the evolution of tooth shape descriptors.

\section{Model for lemur dietary evolution}

Reconstructions of historical dietary ecology and molar morphology allow the tracing of a more complex model for lemur dietary evolution (Fig. 3, 5). The ancestral lemuriform appears to have resembled ancestral lorisiforms and fossil stem strepsirrhines in its molar morphology, and likely pursued a dietary 
ecology of mixed frugivory, gummivory, and insectivory. Cheirogaleids have continued to occupy this ecospace $(48,49)$. The clade uniting lemurids and indriids (including the subfossil families Archaeolemuridae and "Palaeopropithecidae") substantially modified the ancestral strepsirrhine molar morphology in reducing the strength of the protocristid and opening the talonid and trigonid basins. This tooth morphology approaches a more bilophodont morphology, a configuration that combines "blades" for slicing leaves with "wedges" for forcing open seeds $(49,50)$. The evolution of a more bilophodont morphology at this lemurid + indriid node may indicate an important shift toward a mixed diet of fruits enclosed in hard rinds ("defended" fruits), seeds, and leaves.

At this juncture, lemurids and their sister group of indriids + archaeolemurids took divergent paths. The ancestral lemurid broadly resembles Lemur catta in reconstructed tooth shape, which may provide a model for the ecological origins of the group. L. catta consumes a mixed, seasonally shifting diet of leaves and fruits, including many defended fruits enclosed in hard rinds (51). From this generalist ancestor, two lemurid dietary strategies diverged. Eulemur, Varecia, and the extinct Pachylemur evolved toward specializations on fleshy fruits (52-54). Adaptations toward processing soft fruits include the reduction of the trigonid and expansion of the talonid basin, the reduction of the entoconid, and the incorporation of the entoconid into a continuous buccal crest without a talonid notch. Hapalemur and Prolemur, alternatively, evolved toward folivory, and in particular the exploitation of reedy grasses, through the enclosure of the trigonid and talonid basins in high, thickly developed crests $(55,56)$.

The reconstructed LCA of indriids and archaeolemurids is similar in dental morphology to the LCA of lemurids + indriids and this ancestral form may have exploited a diet broadly similar to Propithecus, combining fruits, seeds, and leaves in seasonally shifting combinations $(54,57-59)$. Members of this clade then appear to diverge in their adaptive strategies. The LCA of archaeolemurids shares adaptations for the exploitation of hard foods with the terminal taxa Archaeolemur and Hadropithecus $(60,61)$. The ancestral indriid may have shifted toward the exploitation of leaves, as indicated by the dietary ecology inferred from the reconstructed dental topography metrics at this node, while retaining adaptations for processing seeds and fruit. Molar features characteristic of indriids are present in this reconstructed ancestor, including a reduced protocristid with an expanded, open trigonid basin approaching the talonid in area, a prominent entoconid with a well-developed talonid notch, and bucco-lingually oriented crests connecting the protoconid and metaconid and entoconid and hypoconid. It is possible that these features evolved in an ancestor for which seed predation was a more important dietary activity than folivory. Highly specialized folivores like Indri, Avahi, and some of the subfossil "palaeopropithecids" arise later within this radiation $(58,62,63)$.

\section{Conclusions}

The shift toward the exploitation of defended plant resources at the origins of the lemurid + indriid clade appears to have facilitated the expansion of these clades into morphospace unoccupied by lorisiforms and cheirogaleids (Fig. 4, 5). The acquisition of this new dietary profile occurred during the Oligocene expansion of modern Malagasy forests hypothesized to have occurred as Madagascar passed from the 
influence of desert latitudes into more equatorial climates $(20,35)$, a period that may also have coincided with the dispersal of lemuriforms to Madagascar as inferred by Gunnell et al. (34), or a recovery from a mass extinction at Eocene-Oligocene boundary driven by a global climate shock (10). It is also possible to view the development of an incipiently bilophodont molar as a key innovation permitting the subsequent diversification of large-bodied (lemurid + indriid) lemurs. Whatever its proximate source, ecological opportunity opened by access to defended plant resources in Malagasy forests appears to have been critical in the ecological diversification of lemurs.

Our interpretation of the diversification dynamics of lemurs across deep time continues to be hampered by the lack of a Cenozoic terrestrial fossil record on Madagascar. It is possible that unrecorded radiations occurred during the evolutionary history of lemurs that generated diversity unaccounted for in models relying on the extant and subfossil faunas and included bursts of clade-wide disparity more consistent with adaptive radiation at the origins of the clade, during the Oligocene, or during some other interval (64). This is particularly pertinent given the evidence for widespread extinctions among mammal taxa likely to have dispersed to Madagascar before the Oligocene (10). The model presented here for the evolutionary history of lemur dietary adaptation, however, appears to capture a plausible dietary adaptive history for the extant clade in lieu of future fossil evidence. Further insight may come from the examination of other aspects of lemur ecology potentially related to the exploitation of closed forest habitats, such as locomotion. Further work is also necessary to disentangle the habitat implications of the initiation of global "icehouse" conditions at the beginning of the Oligocene and of the northward drift of Madagascar across the Eocene and Oligocene, which should have had countervailing effects on temperature and precipitation on the island. This might productively focus on the diversification dynamics and historical ecology of the flora characteristic of the different Malagasy forest zones (35).

Whether lemurs represent an exemplar adaptive radiation relies on the framework of adaptive radiation being considered, which varies among theorists (65). The absence of strong evidence for rapid partitioning of ecological space either at the origins of the lemuriform clade or among the lemur clades that diverge during the Oligocene may suggest a model more like that of Simpson's "progressive occupation of numerous zones" by the evolving lemur fauna over the course of the Tertiary (13). However, it seems quite likely that a shift toward the exploitation of defended plant foods during an Oligocene interval of ecological opportunity was critical in driving the taxonomic and ecological diversification of the extant and recently extinct lemur genera.

\section{Methods And Materials}

\section{Sample}

Analyses used digital meshes of second lower molars created from microCT scans of 298 specimens (Supplemental Table 1). This sample includes species from every extant strepsirrhine genus except for the poorly studied Allocebus, seven recently extinct subfossil lemurs, including species from every genus but Archaeoindris, and fossil primate species of the following genera: Adapis, Anchomomys, Cantius, 
Djebelemur, Donrussellia, Karanisia, Komba, Nycticeboides, Plesiopithecus, Pronycticebus, Propotto, Teilhardina, and Wadilemur. Extant taxa were classified to one of three dietary ecologies (frugivory, folivory, or insectivory) based on the largest component of their diet in field studies of wild populations (Supplemental Table 2). Scan processing followed procedures described in the supplemental methods.

\section{Tooth shape}

Functional adaptation to diet is quantified using three dental topography metrics: the sum (here called DNE) and the coefficient of variation (here called DNE CV) of Dirichlet normal energy calculated across a tooth and relief index (RFI). DNE describes the curvature of the occlusal surface as deviation in normal energy from a plane (66). DNE captures tooth sharpness, which, like shearing crest length, is associated with the processing of tough structural carbohydrates. DNE is calculated using the newest "ariaDNE" implementation, which averages vertex-by-vertex DNE across a local area determined using a bandwidth parameter (67). Here the bandwidth parameter is set to 0.10 , which indicates a local influence on each vertex calculation equivalent to $10 \%$ of the tooth surface. RFI is a ratio of a tooth's surface area to its twodimensional projection into an "occlusal plane" $(68,69)$. Occlusal area is measured across the digitized tooth crown cropped at the enamel-cementum junction (sensu Boyer (69)). The combination of DNE, DNE $\mathrm{CV}$, and RFI is effective at distinguishing folivorous from insectivorous strepsirrhines (70), which has previously proved challenging for tooth shape descriptors in the absence of body size information $(66,71,72)$. This allows evolution of functional aspects of tooth shape to be modelled without a potentially confounding consideration of body size evolution.

\section{Phylogeny}

Analyses used the total evidence phylogeny inferred by Herrera and Dávalos (29), which was produced by combining morphological observations on 85 extant and 33 extinct taxa and DNA from 114 extant and 8 extinct (subfossil) taxa, with fossil taxa placed into the tree using a fossilized birth-death process. The topology was modified to reflect the placement of Plesiopithecus and Propotto as sequential sister taxa to Daubentonia in the recent total evidence phylogeny presented by Gunnell et al. (34). Taxa for which no data were available were pruned from the topology and species were collapsed to genera for analysis. Analysis at the genus level avoids issues arising from the reconciliation of changing understandings of lemur alpha taxonomy with data collected from museum specimens and from the uneven representation of specific diversity within genera; maximizes the sample size at each tip state; and allows questions about the differentiation of lemur morphotypes to be addressed without considering the lower-level divergence of lemur species, which may result from processes of allopatric speciation related to Quaternary climate fluctuations (40).

\section{Macroevolutionary model fitting}

The fits of alternate models of evolution were tested for the three dental topography metrics. Adaptive radiation in lemurs, either at the origins of the clade or among the taxa diverging in the Oligocene, would be most consistent with an "early burst" (EB) model, with elevated rates of evolution early in the history of a clade (43). EB models were tested against a null model of Brownian motion (BM) evolution and a 
single-optimum Ornstein-Uhlenbeck (OU) process, which models evolution using an optimum value and an attraction parameter. OU processes have been interpreted as modelling stabilizing selection or as attraction to an adaptive peak, but can also model evolutionary processes in which less phylogenetic signal is present in the distribution of a character state than would be expected by Brownian motion due to high rates of evolution toward the tips of the tree $(46,73,74)$. Model fitting was performed using the package "geiger" in R and fits were compared using the "widely applicable information criterion" (WAIC) $(75,76)$.

\section{Disparity through time}

Subclade partitioning of disparity was assessed using disparity-through-time (DTT) analysis and the calculation of the morphological disparity index (MDI), implemented in "geiger" $(47,75)$. This method calculates the relative partitioning of morphological disparity within and among subclades of a larger clade. Adaptive radiations should show relatively more morphological differentiation among early diverging subclades than within subclades, which can be visualized using a plot of the accumulation of disparity-through-time (DTT) from the root to the tips of a phylogeny. The deviation of the empirical accumulation of subclade disparity from the expected curve under a Brownian motion process is calculated by simulating BM evolution over 10,000 iterations. The difference in area under the empirical DTT and simulated DTT curves is calculated as the MDI. DTT curves and MDI are calculated using the squared Euclidean distances of all three dental topography metrics considered simultaneously.

\section{Rates of evolution through time}

Rates of per-edge change in tooth shape were calculated between adjacent nodes and between nodes and adjacent tips of the lemuriform subclade. This approach allows pulses of morphological differentiation not corresponding to the origins of major clades to be identified and tested for correspondence with intervals of hypothesized ecological opportunity $(77,78)$.

Ancestral state reconstructions (ASR) were performed for each of the three dental topography metrics (DNE, DNE CV, and RFI) in a Bayesian framework using the package 'BayesTraits' (Supplemental material). The means of the reconstructed metrics at each node were then combined in two ways to produce two outputs (here termed "mean reconstructions" = MRs and "posterior distribution of reconstructions" = PDRs). A discriminant function analysis (DFA) model was constructed, trained using extant strepsirrhines of known dietary ecology (Supplemental Table 2). The mean reconstructed values at each node were classified using this DFA model, generating vectors of the relative probabilities that the ancestors at each node could be classified into each of three dietary ecologies (frugivory, folivory, and insectivory) (MRs). This DFA model was also applied to a posterior distribution of 1000 reconstructions created using the complete posterior distributions of reconstructed dental topography metrics (PDRs). Estimating diet over a posterior distribution of reconstructed dental topography metrics incorporates uncertainty from both the DFA estimation of dietary ecology and from the ancestral state reconstructions of the dental topography metrics themselves. The outputs of both MR and PDR models are relative 
probabilities corresponding to each node and reflecting the modelled likelihood of membership in each of the three dietary ecologies by a hypothetic ancestor at that node.

Rates of change in the reconstructed probabilities that ancestral nodes represented folivores were then calculated across all of the edges in the lemur tree by taking the log difference in probabilities between adjacent nodes (or nodes and tips) and dividing by the intervening branch length, a modification of the darwin as a unit of evolutionary rate (79-81). Rates of evolution were calculated from both the single set of reconstructions calculated using the MRs of each node and across the 1000 iteration PDRs. Rate of change in folivory was chosen for analysis because the ancestral lemur was reconstructed as strongly frugivorous, and the relative shift toward folivory should capture the divergent acquisition of adaptations for the exploitation of defended plant resources in newly accessible or expanding forests. Calculating the rate of change in probability estimates allowed the morphological information from the three reconstructed dental topography metrics to be considered together, re-encoded in the context of biologically meaningful information on dietary ecology in strepsirrhines (in contrast to using, for example, the first principal component of a PCA).

The effects of hypothesized intervals of ecological opportunity on rates of evolution were tested using hierarchically structured Bayesian models constructed using the R package 'brms' (Supplemental material). Time bins of 1-million-year duration were created spanning from the initial divergence of lemurs until the present. The rates of evolution across the branches overlapping each 1 million-year time bin were assembled into vectors. Models were constructed to test whether bins occurring within the $10 \mathrm{Ma}$ of the Eocene after the initial divergence of lemurs (48-38 Ma) or during the first $10 \mathrm{Ma}$ of the Oligocene (34-24 Ma) show higher rates of evolution than during other intervals. This is operationally similar to ANOVA, but allows for the hierarchically clustering of rates from different BayesTraits model runs. Tests are done using both only the MRs and using the PDRs aggregated by BayesTraits model run (each of the 1000 sampled iterations from the ancestral state reconstructions). Calculated rates of evolution are expected to rise artificially in branches terminating in extant taxa $(46,78)$. This is comparable to the "Sadler effect" observed by stratigraphers $(46,82)$. In effect, extant taxa haven't "finished evolving," and so have shorter branches than expected by their degree of morphological differentiation. To avoid this issue, observations over the last $10 \mathrm{Ma}$ are excluded.

\section{Ancestral shape reconstruction}

Shape evolution is examined using a novel application of ancestral state reconstruction to continuous, landmark-free representations of 3D shape to generate digital mesh objects representing the ancestral morphologies characterizing nodes on a phylogenetic tree of extant and recently extinct subfossil lemurs. Mesh files representing the teeth of tip taxa are first aligned using Auto3dgm and then registered using a preliminary version of SAMS, an open-source software suite improving on continuous Procrustes methods (83-87). Ancestral shapes are computed using the weighted means of the positions of each digital model vertex using squared changed parsimony (equivalent to a Brownian Motion model of evolution in a maximum likelihood framework [88]) across branches of the tree, a modification of approaches to calculating mean shape using the positions of a set of homologous landmarks (89). 
Models fit to the evolution of DNE and RFI best supported a BM process, suggesting that this may accurately approximate the evolution of tooth shape. Future refinements of this method for reconstructing mean shapes will explore methods for fitting more complex evolutionary models. DNE and DNE CV are then calculated from reconstructed meshes to examine trends in dietary adaptation at internal nodes.

\section{Declarations}

\section{Acknowledgements}

This paper emerged from a dissertation chapter to which Gregg Gunnell, Richard Kay, Daniel McShea, Elizabeth St. Clair, Christine Wall, and Blythe Williams gave helpful guidance. Many undergraduates assisted in processing scans, especially Katrina Montane and Miles Schaeffer. Laurie Godfrey, Eric Seiffert, and the collections staff at the Smithsonian National Museum of Natural History, the American Museum of Natural History, the Field Museum of Natural history, the Natural History Museum (UK), and the Division of Fossil Primates at the Duke Lemur Center all provided valuable access to specimens.

\section{Author contributions}

ELF conceived of the research question and collected the data; ELF, SS, JMW, HK, RR, and SK developed new analytical approaches and analyzed the data.

\section{Funding}

Funding was provided by the Duke Graduate School dissertation research domestic travel grant and summer research support and by the grants NSF BCS 1552848, NSF BCS 130405, and NSF BCS 1825129.

\section{Availability of Data and Materials}

Scanned teeth are archived on the web database Morphosource, with doi provided in the supplemental material.

\section{Ethics Approval and Consent to Participate}

Not applicable.

\section{Consent for Publication}

Not applicable.

\section{Competing Interests}

The authors declare that they have no competing interests. 


\section{References}

1. Godfrey LR, Jungers WL, Schwartz GT. Ecology and extinction of Madagascar's subfossil lemurs. In: Lemurs. Springer; 2006. p. 41-64.

2. Rylands $A B$, Mittermeier RA. Primate taxonomy: species and conservation. Evol Anthrop. 2014;23(1):8-10.

3. Godfrey LR. Subfossil lemurs. In: The International Encyclopedia of Primatology. 2017. p. 1-5.

4. Seiffert ER. Early evolution and biogeography of lorisiform strepsirrhines. Am J Primatol. 2007;69(1):27-35.

5. Martin RD. Review lecture: Adaptive radiation and behaviour of the Malagasy lemurs. Philos T R Soc B. 1972;264(862):295-352.

6. Jungers WL. Adaptive diversity in subfossil Malagasy prosimians. Z Morphol Anthropol. 1980;17786.

7. Wright PC. Lemur traits and Madagascar ecology: coping with an island environment. Am. J Phys Anthropol. 1999;110(S29):31-72.

8. Scott JR, Godfrey LR, Jungers WL, Scott RS, Simons EL, Teaford MF, et al. Dental microwear texture analysis of two families of subfossil lemurs from Madagascar. J Hum Evol. 2009;56(4):405-16.

9. Herrera JP. Testing the adaptive radiation hypothesis for the lemurs of Madagascar. Roy Soc Open Sci. 2017;4(1):161014.

10. Godfrey LR, Samonds KE, Baldwin JW, Sutherland MR, Kamilar JM, Allfisher KL. Mid-Cenozoic climate change, extinction, and faunal turnover in Madagascar, and their bearing on the evolution of lemurs. BMC Evol Biol. 2020;20(1):1-18.

11. Osborn HF. The law of adaptive radiation. Am Nat. 1902;36(425):353-63.

12. Simpson GG. Tempo and mode in evolution. Columbia University Press; 1944.

13. Simpson GG. Major features of evolution. Columbia University Press: New York; 1955.

14. Schluter D. The ecology of adaptive radiation. OUP Oxford; 2000.

15. Gavrilets S, Losos JB. Adaptive radiation: contrasting theory with data. Science. 2009;323(5915):732-7.

16. Losos JB. Adaptive radiation, ecological opportunity, and evolutionary determinism: American Society of Naturalists EO Wilson Award address. Am Nat. 2010;175(6):623-39.

17. Yoder JB, Clancey E, Des Roches S, Eastman JM, Gentry L, Godsoe W, et al. Ecological opportunity and the origin of adaptive radiations. J Evol Biol. 2010;23(8):1581-96.

18. Stroud JT, Losos JB. Ecological opportunity and adaptive radiation. Ann Rev Ecol Evol Syst. 2016;47:507-32.

19. Reed KE, Fleagle JG. Geographic and climatic control of primate diversity. P Natl Acad Sci. 1995;92(17):7874-6. 
20. Wells NA. Some hypotheses on the Mesozoic and Cenozoic paleoenvironmental history of Madagascar. The natural history of Madagascar. 2003;16-34.

21. Ali JR, Huber M. Mammalian biodiversity on Madagascar controlled by ocean currents. Nature. 2010;463(7281):653.

22. Samonds KE, Godfrey LR, Ali JR, Goodman SM, Vences M, Sutherland MR, et al. Spatial and temporal arrival patterns of Madagascar's vertebrate fauna explained by distance, ocean currents, and ancestor type. P Natl Acad Sci. 2012;109(14):5352-7.

23. Samonds KE, Godfrey LR, Ali JR, Goodman SM, Vences M, Sutherland MR, et al. Imperfect isolation: factors and filters shaping Madagascar's extant vertebrate fauna. PLoS One. 2013;8(4):e62086.

24. Millot J. La faune malgache et le mythe gondwanien. Institut scientifique de Madagascar; 1952.

25. Yoder $A D$, Cartmill $M$, Ruvolo $M$, Smith $K$, Vilgalys R. Ancient single origin for Malagasy primates. $P$ Nat Acad Sci. 1996;93(10):5122-6.

26. Garbutt N. Mammals of Madagascar: a complete guide. Yale University Press; 2007.

27. Perelman P, Johnson WE, Roos C, Seuánez HN, Horvath JE, Moreira MA, et al. A molecular phylogeny of living primates. PLoS Genetics. 2011;7(3):e1001342.

28. Kistler L, Ratan A, Godfrey LR, Crowley BE, Hughes CE, Lei R, et al. Comparative and population mitogenomic analyses of Madagascar's extinct, giant 'subfossil'lemurs. J Hum Evol. 2015;79:45-54.

29. Herrera JP, Dávalos LM. Phylogeny and divergence times of lemurs inferred with recent and ancient fossils in the tree. Syst Biol. 2016;65(5):772-91.

30. Yoder AD, Yang Z. Estimation of primate speciation dates using local molecular clocks. Mol Biol Evol. 2000;17(7):1081-90.

31. Horvath JE, Weisrock DW, Embry SL, Fiorentino I, Balhoff JP, Kappeler P, et al. Development and application of a phylogenomic toolkit: resolving the evolutionary history of Madagascar's lemurs. Genome Res. 2008;18(3):489-99.

32. Chatterjee HJ, Ho SY, Barnes I, Groves C. Estimating the phylogeny and divergence times of primates using a supermatrix approach. BMC evolutionary biology. 2009;9(1):1-19.

33. Springer MS, Meredith RW, Gatesy J, Emerling CA, Park J, Rabosky DL, et al. Macroevolutionary dynamics and historical biogeography of primate diversification inferred from a species supermatrix. PloS one. 2012;7(11):e49521.

34. Gunnell GF, Boyer DM, Friscia AR, Heritage S, Manthi FK, Miller ER, et al. Fossil lemurs from Egypt and Kenya suggest an African origin for Madagascar's aye-aye. Nature communications. 2018;9(1):3193.

35. Buerki S, Devey DS, Callmander MW, Phillipson PB, Forest F. Spatio-temporal history of the endemic genera of Madagascar. Bot J Linn Soc. 2013;171(2):304-29.

36. Fleagle JG. Primate Adaptation and Evolution. 3rd ed. New York: Academic Press; 2013.

37. Godinot M. Fossil Record of the Primates from the Paleocene to the Oligocene. In: Handbook of paleoanthropology. Springer; 2015. p. 1137-259. 
38. Goodman SM, Ganzhorn JU. Biogeography of lemurs in the humid forests of Madagascar: the role of elevational distribution and rivers. J Biogeog. 2004;31(1):47-55.

39. Wilmé L, Goodman SM, Ganzhorn JU. Biogeographic evolution of Madagascar's microendemic biota. Science. 2006;312(5776):1063-5.

40. Mercier J-L, Wilmé L. The Eco-Geo-Clim model: explaining Madagascar's endemism. Madagascar Conservation \& Development. 2013;8(2):63-8.

41. Glor RE. Phylogenetic insights on adaptive radiation. Ann Rev Ecol Evol Syst. 2010;41:251-70.

42. Slater GJ, Price SA, Santini F, Alfaro ME. Diversity versus disparity and the radiation of modern cetaceans. Proc R Soc B. 2010;277(1697):3097-104.

43. Harmon LJ, Losos JB, Jonathan Davies T, Gillespie RG, Gittleman JL, Bryan Jennings W, et al. Early bursts of body size and shape evolution are rare in comparative data. Evolution. 2010;64(8):238596.

44. Streelman JT, Danley PD. The stages of vertebrate evolutionary radiation. Trends Ecol Evol. 2003;18(3):126-31.

45. Ungar PS. Mammal teeth: origin, evolution, and diversity. JHU Press; 2010.

46. Slater GJ, Friscia AR. Hierarchy in adaptive radiation: A case study using the Carnivora (Mammalia). Evolution. 2019;73(3):524-39.

47. Harmon LJ, Schulte JA, Larson A, Losos JB. Tempo and mode of evolutionary radiation in iguanian lizards. Science. 2003;301(5635):961-4.

48. Hladik CM, Charles-Dominique P, Petter J-J. Feeding strategies of five nocturnal prosimians in the dry forest of the west coast of Madagascar. Nocturnal Malagasy primates: ecology, physiology, and behavior. 1980;41-73.

49. Cuozzo FP, Yamashita N. Impact of ecology on the teeth of extant lemurs: a review of dental adaptations, function, and life history. In: Lemurs. Springer; 2006. p. 67-96.

50. Lucas PW, Teaford MF. Functional morphology of colobine teeth. Colobine monkeys: Their ecology, behaviour and evolution. 1994;173-203.

51. Gould L. Lemur catta ecology: what we know and what we need to know. In: Lemurs. Springer; 2006. p. $255-74$.

52. Vasey N. Niche separation in Varecia variegata rubra and Eulemur fulvus albifrons: I. Interspecific patterns. Am J Phys Anthropol. 2000;112(3):411-31.

53. Vasey N. Niche separation in Varecia variegata rubra and Eulemur fulvus albifrons: II. Intraspecific patterns. Am J Phys Anthropol. 2002;118(2):169-83.

54. Sato H, Santini L, Patel ER, Campera M, Yamashita N, Colquhoun IC, et al. Dietary flexibility and feeding strategies of Eulemur: a comparison with Propithecus. In J Primatol. 2016;37(1):109-29.

55. Overdorff DJ, Strait SG, Telo A. Seasonal variation in activity and diet in a small-bodied folivorous primate, Hapalemur griseus, in southeastern Madagascar. Am J Primatol. 1997;43(3):211-23. 
56. Jernvall J, Gilbert CC, Wright PC. Peculiar tooth homologies of the greater bamboo lemur (Prolemur= Hapalemur simus). In: Elwyn Simons: A Search for Origins. Springer; 2008. p. 335-42.

57. Hemingway CA. Morphology and phenology of seeds and whole fruit eaten by Milne-Edwards' sifaka, Propithecus diadema edwardsi, in Ranomafana National Park, Madagascar. Int J Primatol. 1996;17(5):637-59.

58. Powzyk JA, Mowry CB. Dietary and feeding differences between sympatric Propithecus diadema diadema and Indri indri. Int J Primatol. 2003;24(6):1143-62.

59. Norscia I, Carrai V, Borgognini-Tarli SM. Influence of dry season and food quality and quantity on behavior and feeding strategy of Propithecus verreauxi in Kirindy, Madagascar. Int J Primatol. 2006;27(4):1001-22.

60. Godfrey LR, Semprebon GM, Schwartz GT, Burney DA, Jungers WL, Flanagan EK, et al. New insights into old lemurs: the trophic adaptations of the Archaeolemuridae. Int J Primatol. 2005;26(4):825-54.

61. Godfrey LR, Crowley BE, Muldoon KM, Kelley EA, King SJ, Best AW, et al. What did Hadropithecus eat, and why should paleoanthropologists care? Am J Phys Anthropol. 2016;78(10):1098-112.

62. Thalmann U. Food resource characteristics in two nocturnal lemurs with different social behavior: Avahi occidentalis and Lepilemur edwardsi. Int J Primatol. 2001;22(2):287-324.

63. Britt A, Randriamandratonirina NJ, Glasscock KD, lambana BR. Diet and feeding behaviour of Indri indri in a low-altitude rain forest. Folia Primatologica. 2002;73(5):225-39.

64. Foote M. The evolution of morphological diversity. Ann Rev Ecol Syst. 1997;28(1):129-52.

65. Olson ME, Arroyo-Santos A. Thinking in continua: beyond the "adaptive radiation" metaphor. BioEssays. 2009;31(12):1337-46.

66. Bunn JM, Boyer DM, Lipman Y, St Clair EM, Jernvall J, Daubechies I. Comparing Dirichlet normal surface energy of tooth crowns, a new technique of molar shape quantification for dietary inference, with previous methods in isolation and in combination. Am J Phys Anthropol. 2011;145(2):247-61.

67. Shan S, Kovalsky SZ, Winchester JM, Boyer DM, Daubechies I. aria DNE: A Robustly Implemented Algorithm for Dirichlet Energy of the Normal. Methods Ecol. Evol. 2019;

68. Ungar PS, Williamson M. Exploring the effects of tooth wear on functional morphology: a preliminary study using dental topographic analysis. Palaeontologia Electronica. 2000;3(1):1-18.

69. Boyer DM. Relief index of second mandibular molars is a correlate of diet among prosimian primates and other euarchontan mammals. J Hum Evol. 2008;55(6):1118-37.

70. Fulwood EL. Lemur Teeth in Three Keys: Dietary Adaptation, Ecospace Occupation, and Macroevolutionary Dynamics. Duke University; 2019.

71. Kay RF. The functional adaptations of primate molar teeth. Am J Phys Anthropol. 1975;43(2):195215.

72. Winchester JM, Boyer DM, St Clair EM, Gosselin-Ildari AD, Cooke SB, Ledogar JA. Dental topography of platyrrhines and prosimians: convergence and contrasts. Am J Phys Anthropol. 2014;153(1):2944. 
73. Beaulieu JM, Jhwueng D-C, Boettiger C, O'Meara BC. Modeling stabilizing selection: expanding the Ornstein-Uhlenbeck model of adaptive evolution. Evolution. 2012;66(8):2369-83.

74. Cooper N, Thomas GH, Venditti C, Meade A, Freckleton RP. A cautionary note on the use of Ornstein Uhlenbeck models in macroevolutionary studies. Biol J Linn Soc. 2015;

75. Harmon LJ, Weir JT, Brock CD, Glor RE, Challenger W. GEIGER: investigating evolutionary radiations. Bioinformatics. 2007;24(1):129-31.

76. Watanabe S. A widely applicable Bayesian information criterion. J Mach Learn Res. 2013;14(Mar):867-97.

77. Brusatte SL, Montanari S, Yi H, Norell MA. Phylogenetic corrections for morphological disparity analysis: new methodology and case studies. Paleobiology. 2011;37(1):1-22.

78. Wright DF. Phenotypic innovation and adaptive constraints in the evolutionary radiation of Palaeozoic crinoids. Sci Rep. 2017;7(1):13745.

79. Haldane JBS. Suggestions as to quantitative measurement of rates of evolution. Evolution. 1949;3(1):51-6.

80. Gingerich P. Rates of evolution: effects of time and temporal scaling. Science. 1983;222:159-62.

81. Gingerich PD. Rates of evolution. Ann Rev Ecol Evol Syst. 2009;40:657-75.

82. Sadler PM. Sediment accumulation rates and the completeness of stratigraphic sections. J Geol. 1981;89(5):569-84.

83. Boyer DM, Puente J, Gladman JT, Glynn C, Mukherjee S, Yapuncich GS, et al. A new fully automated approach for aligning and comparing shapes. Anat Rec. 2015;298(1):249-76.

84. Boyer DM, Lipman Y, Clair ES, Puente J, Patel BA, Funkhouser T, et al. Algorithms to automatically quantify the geometric similarity of anatomical surfaces. P Nat Acad Sci. 2011;108(45):18221-6.

85. Gao T, Yapuncich GS, Daubechies I, Mukherjee S, Boyer DM. Development and assessment of fully automated and globally transitive geometric morphometric methods, with application to a biological comparative dataset with high interspecific variation. Anat Rec. 2018;301(4):636-58.

86. Al-Aifari R, Daubechies I, Lipman Y. Continuous Procrustes distance between two surfaces. Commun Pure Appl Math. 2013;66(6):934-64.

87. Ravier RJ. Eyes on the Prize: Improved Registration via Forward Propagation. arXiv preprint arXiv:181210592. 2018.

88. Nunn CL. The comparative approach in evolutionary anthropology and biology. University of Chicago Press; 2011.

89. Wiley DF, Amenta N, Alcantara DA, Ghosh D, Kil YJ, Delson E, et al. Evolutionary morphing. In: VIS 05 IEEE Visualization, 2005. IEEE; 2005. p. 431-8.

\section{Figures}



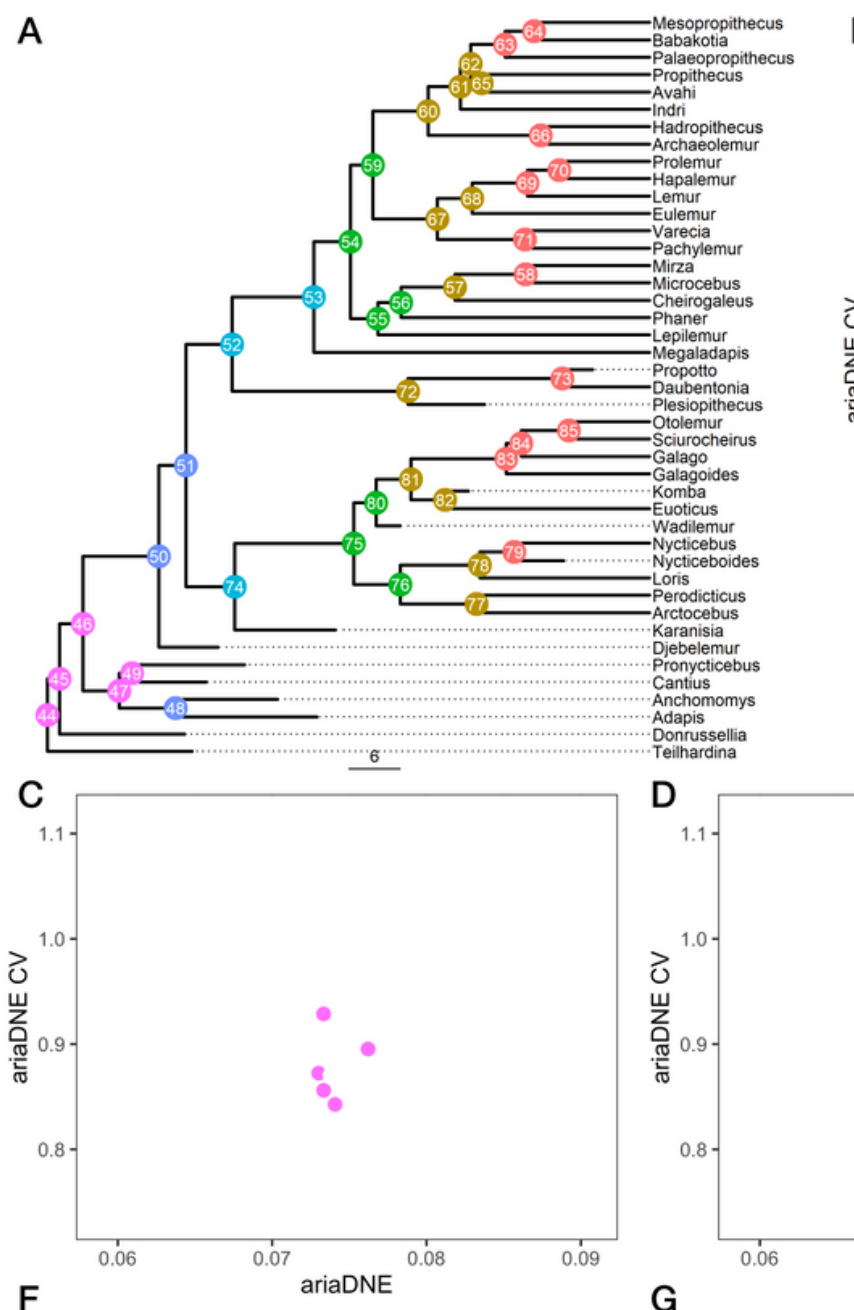

B
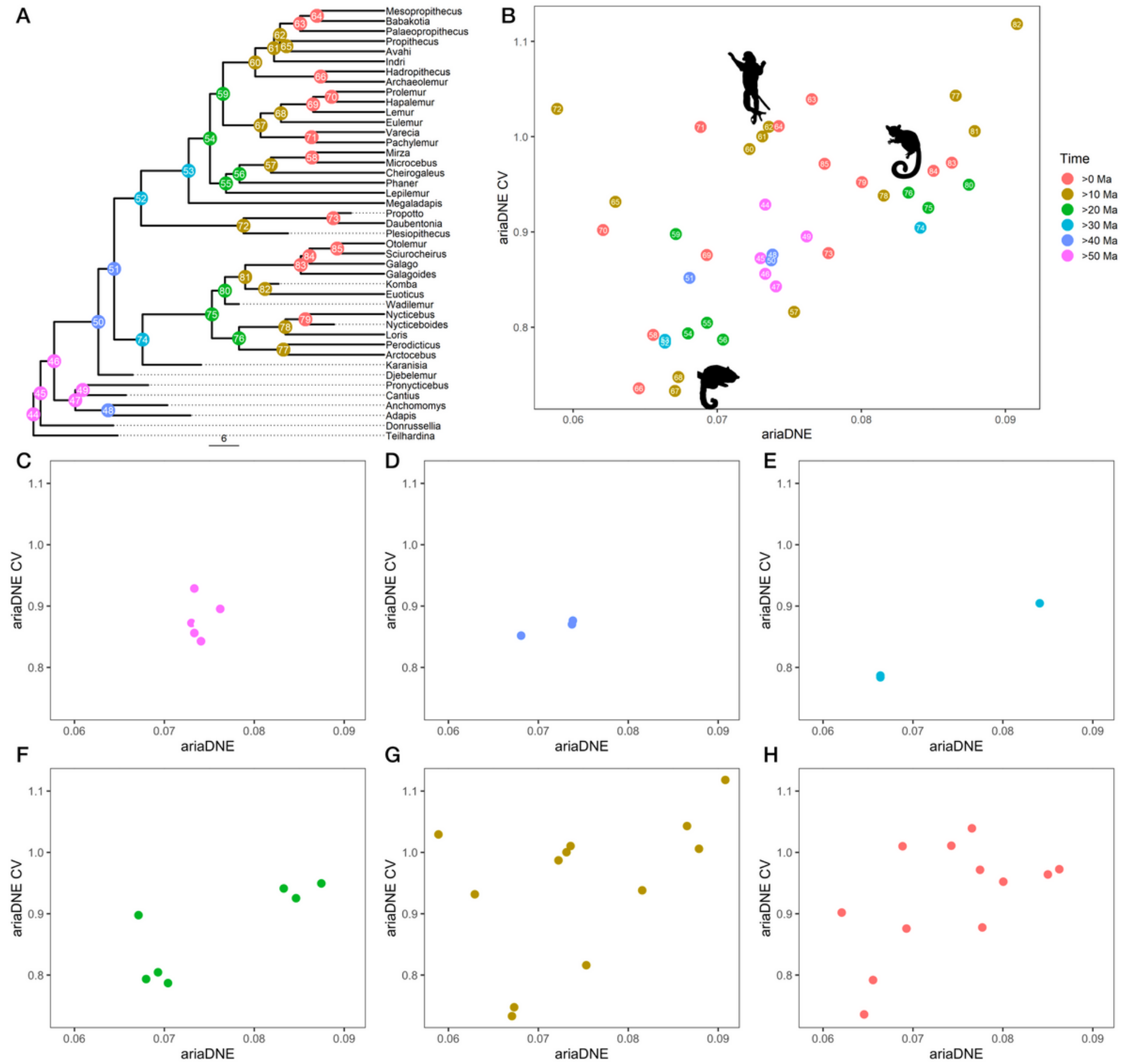

\section{Figure 1}

Reconstructed lower second molar morphology at internal nodes in the tree of strepsirrhines. Green branches represent lemuriforms; blue branches represent lorisiforms; Red branches represent stem strepsirrhines and other fossil primates. Scale bar indicates branch length in million years. 


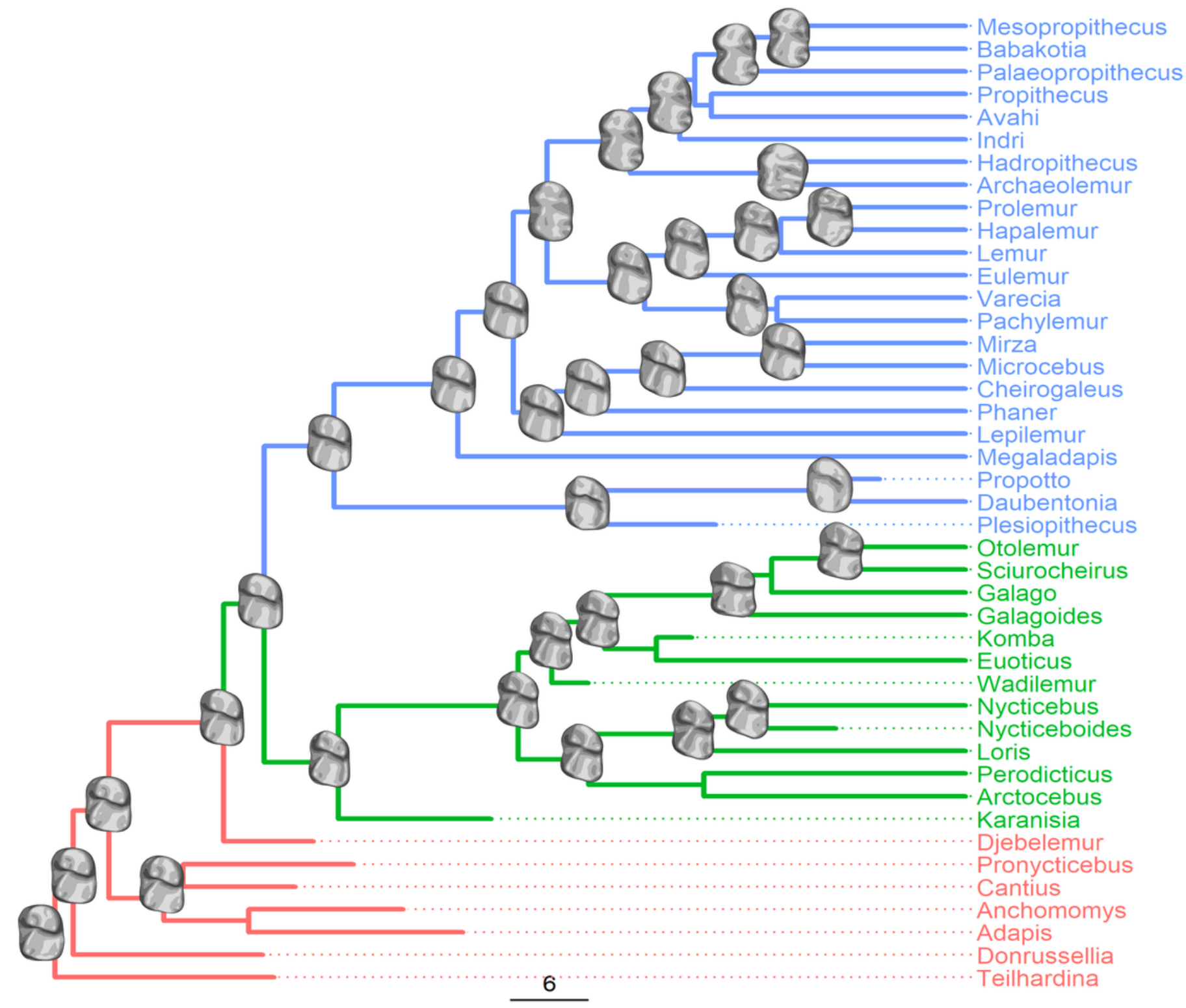

\section{Figure 1}

Hypothesized model for the dietary evolution of lemuriforms drawing from reconstructed lower second molar morphology and dietary ecology inferred from ancestral state reconstructions of dental topography metrics. Dietary states at internal nodes represent hypotheses for the ecologies at ancestors of extant lemur groups. Lepilemur and Megaladapis, which diverge near the origins of lemuriformes and evolve toward folivory, are not figured. 


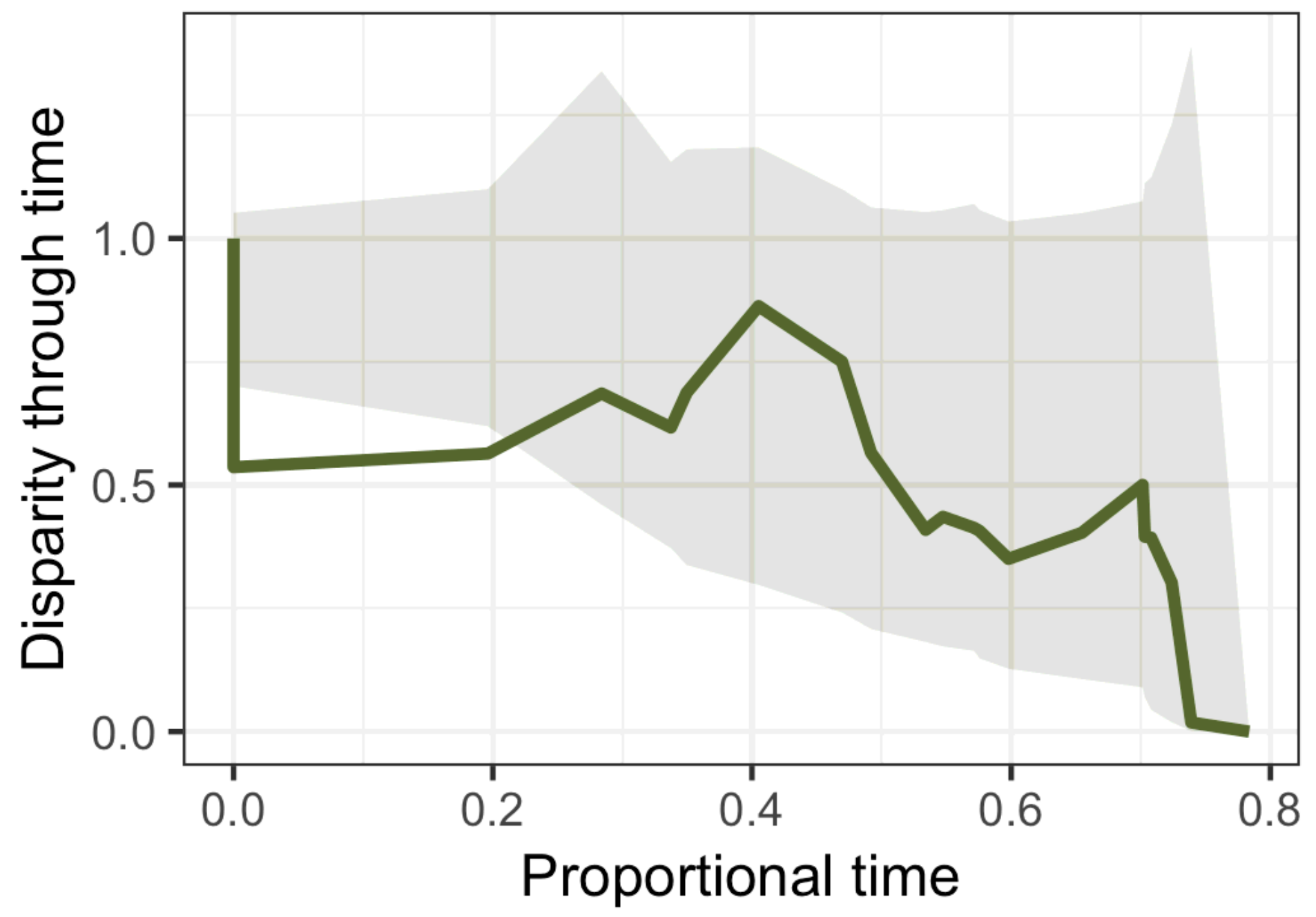

Figure 1

Mean rate evolution of dental adaptations for folivory at each time interval across the tree of Lemuriformes. Solid line indicates the mean rates at each bin of one-million-year duration. Dotted line with grey ribbon indicates LOWESS smoothed trend in the empirical data. The 10 Ma before present shows a uniformly high mean rate of evolution, likely reflecting a "Sadler effect" of apparently rapid evolution in extant branches (Wright, 2017; Slater and Friscia, 2019). 


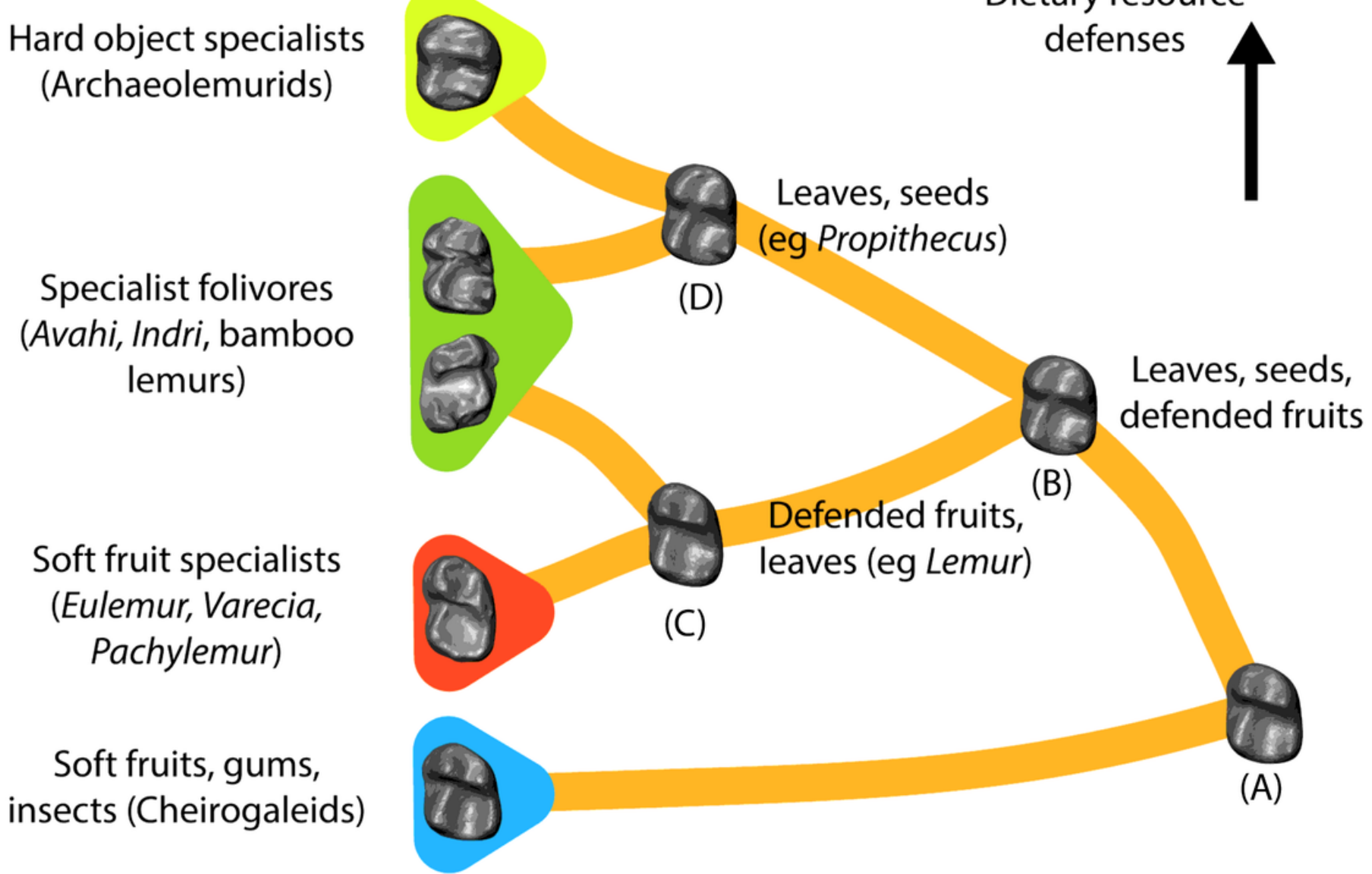

\section{Figure 1}

Disparity through time (DTT) plot of lemurs from the origins of the clade. Shaded region represents $95 \%$ $\mathrm{Cl}$ of disparity modelled under a Brownian Motion process. Solid line indicates empirical disparity. Zero represents the root node of lemuriforms and lorisiforms. The calculation of DTT ends at the divergence of the most recent subclade (approximately $78 \%$ of the time distance until the present) 


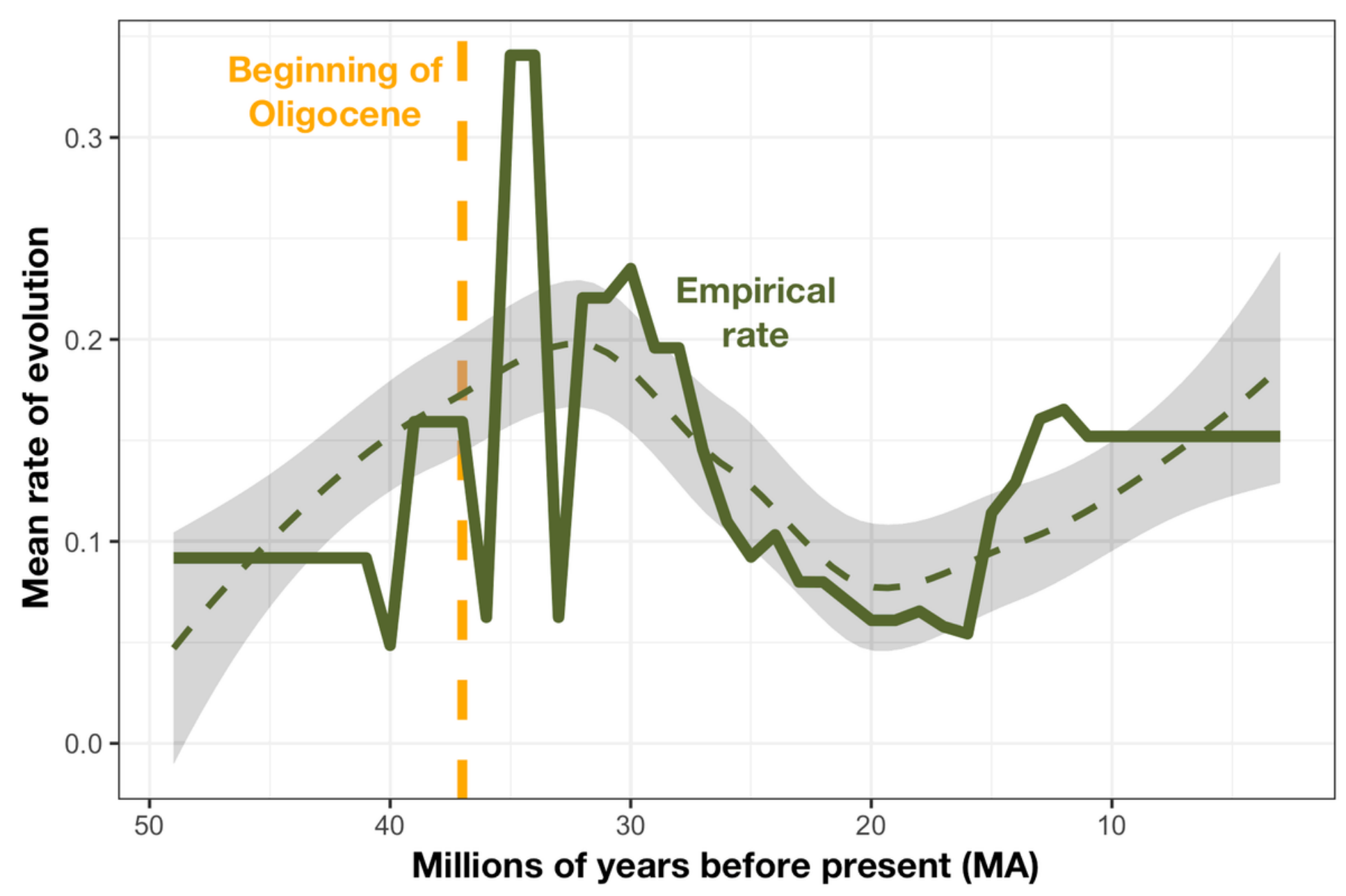

Figure 1

Scatterplot of ariaDNE and ariaDNE CV calculated on reconstructed lower second molar morphology at internal nodes in the tree of strepsirrhines, with node numbers indicated on the phylogeny. Node values are discretized into 10 million-year intervals. Scale bar indicates branch length in million years. A) Position of nodes on phylogeny; B) Scatterplot of nodes from all time intervals. Silhouettes represent approximate regions in which nodes of major clades cluster. Indriids are in the upper center, Lorisiformes in the upper right, and cheirogaleids in the lower left. C-H) Nodes from each time interval plotted separately showing pattern of ecospace expansion.

\section{Supplementary Files}

This is a list of supplementary files associated with this preprint. Click to download.

- Supplementaltext.docx 